\title{
DING PROJECTIVE MODULES WITH RESPECT TO A SEMIDUALIZING BIMODULE
}

\author{
CHUNXIA ZHANG, LIMIN WANG AND ZHONGKUI LIU
}

\begin{abstract}
Let $R$ and $S$ be rings and ${ }_{S} C_{R}$ a faithfully semidualizing bimodule. A left $S$-module $M$ is called Ding $C$-projective if there exists an exact sequence of $C$-projective left $S$-modules

$X=\cdots \rightarrow C \otimes_{R} P_{1} \rightarrow C \otimes_{R} P_{0} \rightarrow C \otimes_{R} P^{0} \rightarrow C \otimes_{R} P^{1} \rightarrow \cdots$ such that $M \cong \operatorname{Coker}\left(C \otimes_{R} P_{1} \rightarrow C \otimes_{R} P_{0}\right)$ and the complexes $\operatorname{Hom}_{S}\left(C \otimes_{R} P, X\right)$ and $\operatorname{Hom}_{S}\left(X, C \otimes_{R} F\right)$ are exact for any projective left $R$-module $P$ and any flat left $R$ module $F$. The properties of Ding $C$-projective modules and dimensions are given. Among others, the Foxby equivalences between some subclasses of the Auslander class and the Bass class are also investigated.
\end{abstract}

Introduction. Throughout this work, $R$ and $S$ are fixed associated rings with identity and all $R$ - and $S$-modules are understood to be unitary left $R$ - and $S$-modules. Right $R$ - and $S$-modules are identified with left modules over the opposite rings $R^{o p}$ and $S^{o p}$. Also, $C={ }_{S} C_{R}$ is a fixed faithfully semidualizing bimodule, cf., Definition 1.1 below.

In basic homological algebra, projective, injective and flat modules play an important and fundamental role. Over a commutative Noetherian ring, Holm and Jørgensen [9] introduced the $C$-Gorenstein projective and $C$-Gorenstein injective modules using semidualizing modules and their associated projective and injective classes, and they connected the study of semidualizing modules to associated Auslander and Bass classes for semidualizing modules, $\mathcal{A}_{C}(R)$ and $\mathcal{B}_{C}(R)$, which are subcategories of the category of $R$-modules. White [17] further considered these modules when $R$ is a commutative ring, and she called $C$-Gorenstein projective modules as $G_{C}$-projectives and $C$-Gorenstein

2010 AMS Mathematics subject classification. Primary 16D40, 16E10.

Keywords and phrases. Semidualizing modules, Ding $C$-projective modules, $G_{C^{-}}$ projective modules, Auslander categories.

Research supported by National Natural Science Foundation of China (Nos. 11261050, 11301240, 11401475) and by NWNU-lkqn-12-17.

Received by the editors on April 19, 2012, and in revised form on April 13, 2013. 
injective modules as $G_{C}$-injectives. In particular, many results about the Gorenstein projectivity and Gorenstein injectivity in $[\mathbf{3}, \mathbf{8}, \mathbf{1 0}]$ were generalized in [17]. Recently, Liu et al. [11] extended these modules to arbitrary associative rings; also, they have proved that many results for Auslander and Bass classes associated to a semidualizing module over a commutative Noetherian ring can be extended to arbitrary associative rings.

For a faithfully semidualizing bimodule ${ }_{S} C_{R}$, denote by $\mathcal{G P}(R)$, $\mathcal{G I}(S), \mathcal{G P}_{C}(S)$ and $\mathcal{G I}_{C}(R)$ the classes of Gorenstein projective, Gorenstein injective, $G_{C}$-projective and $G_{C}$-injective modules, respectively. From [11, Theorem 4.6], we have the following Foxby equivalence diagram:

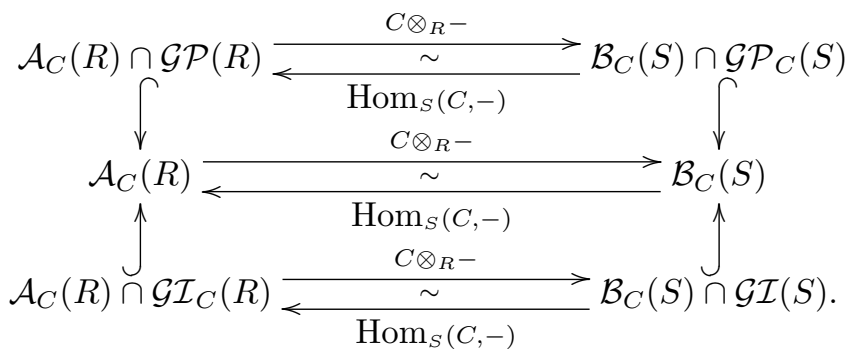

On the other hand, Ding et al. $[\mathbf{2}, \mathbf{1 3}]$ considered two special cases of the Gorenstein projective and Gorenstein injective modules using projective, flat classes and injective, FP-injective classes, which they called strongly Gorenstein flat and Gorenstein FP-injective modules, respectively. The same modules were studied by Gillespie [6] with the different names: Ding projective and Ding injective modules, respectively. By $\mathcal{D} \mathcal{P}(R)$ and $\mathcal{D} \mathcal{I}(S)$, we denote the classes of Ding projective and Ding injective modules, respectively. Thus, for a faithfully semidualizing bimodule ${ }_{S} C_{R}$, a natural question arises: What are the counterparts to the Ding projective and Ding injective modules under Foxby equivalence? So, the motivation of this article is the "?" and 
"??" in the following Foxby equivalence diagram:

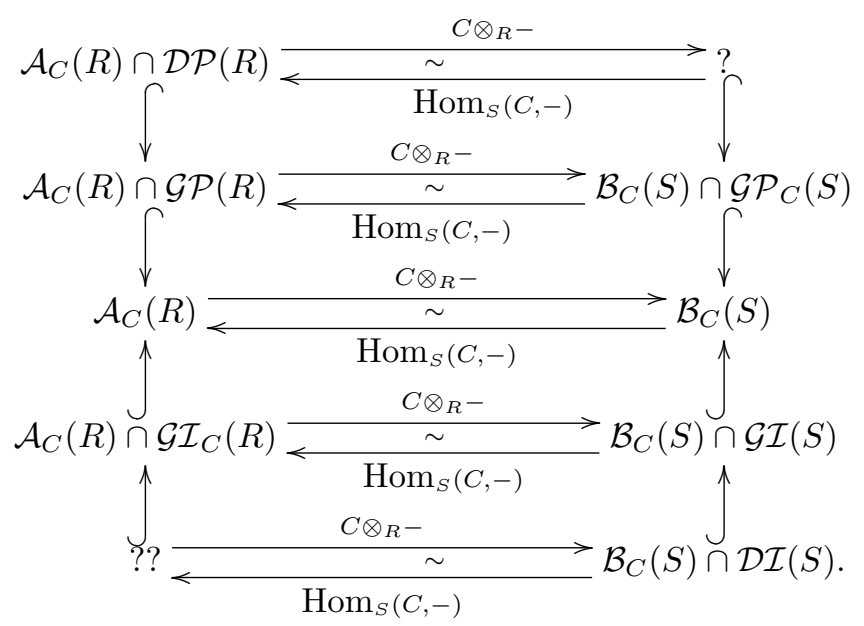

We shall introduce the notions of Ding $C$-projective and Ding $C$ injective modules which play the roles of "?" and "??".

In Section 2, we define and study Ding $C$-projective and Ding $C$ injective modules. An $S$-module $M$ is called Ding $C$-projective if there exists an exact sequence of $C$-projective $S$-modules

$X=\cdots \longrightarrow C \otimes_{R} P_{1} \longrightarrow C \otimes_{R} P_{0} \longrightarrow C \otimes_{R} P^{0} \longrightarrow C \otimes_{R} P^{1} \longrightarrow \cdots$

such that $M \cong \operatorname{Coker}\left(C \otimes_{R} P_{1} \rightarrow C \otimes_{R} P_{0}\right)$ and the complexes $\operatorname{Hom}_{S}\left(C \otimes_{R} P, X\right)$ and $\operatorname{Hom}_{S}\left(X, C \otimes_{R} F\right)$ are exact for any projective $R$-module $P$ and any flat $R$-module $F$. An $R$-module $N$ is called $D i n g$ $C$-injective if there exists an exact sequence of $C$-injective $R$-modules

$$
\begin{aligned}
X=\cdots \longrightarrow \operatorname{Hom}_{S}\left(C, I_{1}\right) & \longrightarrow \operatorname{Hom}_{S}\left(C, I_{0}\right) \\
& \longrightarrow \operatorname{Hom}_{S}\left(C, I^{0}\right) \longrightarrow \operatorname{Hom}_{S}\left(C, I^{1}\right) \longrightarrow \cdots
\end{aligned}
$$

such that $N \cong \operatorname{Coker}\left(\operatorname{Hom}_{S}\left(C, I_{1}\right) \rightarrow \operatorname{Hom}_{S}\left(C, I_{0}\right)\right)$ and the complexes $\operatorname{Hom}_{R}\left(X, \operatorname{Hom}_{S}(C, I)\right)$ and $\operatorname{Hom}_{R}\left(\operatorname{Hom}_{S}(C, E), X\right)$ are exact for any injective $S$-module $I$ and any FP-injective $S$-module $E$. We prove that the class of Ding $C$-projective (Ding $C$-injective) modules is closed under extensions and direct summands.

Section 3 is devoted to investigating the Ding $C$-projective (Ding $C$-injective) dimensions of modules. We give functorial descriptions of 
these dimensions in Theorem 3.3.

In Section 4, we prove that the subcategories of Ding projective $R$ modules in the Auslander class $\mathcal{A}_{C}(R)$ (Ding injective $S$-modules in the Bass class $\left.\mathcal{B}_{C}(S)\right)$ and Ding $C$-projective $S$-modules (Ding $C$-injective $R$-modules) are equivalent under Foxby equivalence.

1. Preliminaries. In this section, we shall recall some notions and definitions which we need in the later sections.

The study of semidualizing modules over commutative Noetherian rings was initiated independently (with different names) by Foxby [4], Golod [7], and Vasconcelos [16].

Definition 1.1. Let $R$ and $S$ be rings. Following [10], an $(S, R)$ bimodule ${ }_{S} C_{R}$ is semidualizing if

(1) ${ }_{S} C$ admits a degreewise finite $S$-projective resolution.

(2) $C_{R}$ admits a degreewise finite $R^{o p}$-projective resolution.

(3) The homothety map $S_{S} \stackrel{s \gamma}{\rightarrow} \operatorname{Hom}_{R}(C, C)$ is an isomorphism.

(4) The homothety map $R_{R} R_{R} \stackrel{\gamma_{R}}{\rightarrow} \operatorname{Hom}_{S}(C, C)$ is an isomorphism.

(5) $\operatorname{Ext}_{S}^{\geqslant 1}(C, C)=0$.

(6) $\operatorname{Ext}_{R^{o p}} \geqslant 1(C, C)=0$.

A semidualizing bimodule ${ }_{S} C_{R}$ is faithfully semidualizing if it satisfies the following conditions for all modules ${ }_{S} N$ and $M_{R}$ :

(1) If $\operatorname{Hom}_{S}(C, N)=0$, then $N=0$.

(2) If $\operatorname{Hom}_{R^{o p}}(C, M)=0$, then $M=0$.

Let $C={ }_{S} C_{R}$ be a semidualizing bimodule. We set $\mathcal{P}_{C}(S)$ to be the subcategory of modules $C \otimes_{R} P$ where $P$ is $R$-projective, $\mathcal{F}_{C}(S)$ to be the subcategory of modules $C \otimes_{R} F$ where $F$ is $R$-flat, $\mathcal{I}_{C}(R)$ to be the subcategory of modules $\operatorname{Hom}_{S}(C, I)$ where $I$ is $S$-injective and $\mathcal{F I}_{C}(R)$ to be the subcategory of modules $\operatorname{Hom}_{S}(C, E)$ where $E$ is $S$ FP-injective. Modules in $\mathcal{P}_{C}(S), \mathcal{F}_{C}(S), \mathcal{I}_{C}(R)$ and $\mathcal{F} \mathcal{I}_{C}(R)$ are called $C$-projective, $C$-flat, $C$-injective and $C$-FP-injective, respectively. By setting ${ }_{S} C_{R}={ }_{R} R_{R}$ in the definitions above we see that $\mathcal{P}_{R}(R), \mathcal{F}_{R}(R)$, $\mathcal{I}_{R}(R)$ and $\mathcal{F} \mathcal{I}_{R}(R)$ are the classes of ordinary projective, flat, injective and FP-injective $R$-modules, respectively, which we denote by $\mathcal{P}(R)$, $\mathcal{F}(R), \mathcal{I}(R)$ and $\mathcal{F} \mathcal{I}(R)$. 
Note that all semidualizing modules are faithfully semidualizing over a commutative ring (see [10, Proposition 3.1]).

Definition 1.2. Let ${ }_{S} C_{R}$ be a semidualizing bimodule.

The Auslander class $\mathcal{A}_{C}(R)$ with respect to $C$ consists of all $R$ modules $M$ satisfying

(1) $\operatorname{Tor}_{\geqslant 1}^{R}(C, M)=0=\operatorname{Ext}_{S}^{\geqslant 1}\left(C, C \otimes_{R} M\right)$, and

(2) the natural evaluation homomorphism $\mu_{M}: M \rightarrow \operatorname{Hom}_{S}\left(C, C \otimes_{R}\right.$ $M)$ is an isomorphism (of $R$-modules).

The Bass class $\mathcal{B}_{C}(S)$ with respect to $C$ consists of all $S$-modules $N$ satisfying

(1) $\operatorname{Ext}_{S}^{\geqslant 1}(C, N)=0=\operatorname{Tor}_{\geqslant 1}^{R}\left(C, \operatorname{Hom}_{S}(C, N)\right)$, and

(2) the natural evaluation homomorphism $\nu_{N}: C \otimes_{R} \operatorname{Hom}_{S}(C, N) \rightarrow$ $N$ is an isomorphism (of $S$-modules).

The following notions were introduced by Holm and Jørgensen [9] over commutative Noetherian rings and by White [17] for commutative rings.

Definition 1.3. A complete $\mathcal{P} \mathcal{P}_{C}$-resolution is a complex $X$ of $R$ modules satisfying the following conditions:

(1) $X$ is exact and $\operatorname{Hom}_{R}\left(-, \mathcal{P}_{C}(R)\right)$-exact; and

(2) $X_{i}$ is projective for $i \geq 0$ and $X_{i}$ is $C$-projective for $i<0$.

An $R$-module $M$ is $G_{C}$-projective if there exists a complete $\mathcal{P} \mathcal{P}_{C^{-}}$ resolution $X$ such that $M \cong \operatorname{Coker}\left(\partial_{1}^{X}\right)$, and in this case $X$ is called a complete $\mathcal{P} \mathcal{P}_{C}$-resolution of $M$. Set

$$
\mathcal{G} \mathcal{P}_{C}(R)=\text { the subcategory of } \mathrm{G}_{C} \text {-projective } R \text {-modules. }
$$

In the case $C=R$ we use the more common terminology "complete projective resolution" and "Gorenstein projective module" and the notation $\mathcal{G P}(R)$.

A complete $\mathcal{I}_{C} \mathcal{I}$-resolution is a complex $Y$ of $R$-modules such that:

(1) $Y$ is exact and $\operatorname{Hom}_{R}\left(\mathcal{I}_{C}(R),-\right)$-exact; and

(2) $Y_{i}$ is injective for $i \leq 0$ and $Y_{i}$ is $C$-injective for $i>0$. 
An $R$-module $N$ is $G_{C}$-injective if there exists a complete $\mathcal{I}_{C} \mathcal{I}$ resolution $Y$ such that $N \cong \operatorname{Ker}\left(\partial_{0}^{Y}\right)$, and in this case $Y$ is called a complete $\mathcal{I}_{C} \mathcal{I}$-resolution of $N$. Set

$$
\mathcal{G I}_{C}(R)=\text { the subcategory of } \mathrm{G}_{C} \text {-injective } R \text {-modules. }
$$

In the case $C=R$, we use the more common terminology "complete injective resolution" and "Gorenstein injective module" and the notation $\mathcal{G} \mathcal{I}(R)$.

Definition 1.4. An $R$-module $M$ is called Ding projective if there exists an exact sequence of projective $R$-modules

$$
\cdots \longrightarrow P_{1} \longrightarrow P_{0} \longrightarrow P^{0} \longrightarrow P^{1} \longrightarrow \cdots
$$

with $M=\operatorname{Coker}\left(P_{1} \rightarrow P_{0}\right)$ and which remains exact after applying $\operatorname{Hom}_{R}(-, \mathcal{F}(R))$. Set

$\mathcal{D P}(R)=$ the subcategory of Ding projective $R$-modules.

An $S$-module $N$ is called Ding injective if there exists an exact sequence of injective $S$-modules

$$
\cdots \longrightarrow I_{1} \longrightarrow I_{0} \longrightarrow I^{0} \longrightarrow I^{1} \longrightarrow \cdots
$$

with $N=\operatorname{Coker}\left(I_{1} \rightarrow I_{0}\right)$ and which remains exact after applying $\operatorname{Hom}_{S}(\mathcal{F} \mathcal{I}(S),-)$. Set

$$
\mathcal{D I}(S)=\text { the subcategory of Ding injective } S \text {-modules. }
$$

Note that every Ding projective (respectively, Ding injective) module is Gorenstein projective (respectively, Gorenstein injective), and if $R$ is Gorenstein, then every Gorenstein projective (respectively, Gorenstein injective) module is Ding projective (respectively, Ding injective).

Definition 1.5. Let $\mathcal{X}$ be a class of $R$-modules and $M$ an $R$-module. An $\mathcal{X}$-resolution of $M$ is a complex of $R$-modules in $\mathcal{X}$ of the form

$$
X=\cdots \longrightarrow X_{n} \longrightarrow X_{n-1} \longrightarrow \cdots \longrightarrow X_{1} \longrightarrow X_{0} \longrightarrow 0
$$

such that $\mathrm{H}_{0}(X) \cong M$ and $\mathrm{H}_{n}(X)=0$ for $n \geq 1$, and the following exact sequence is the augmented $\mathcal{X}$-resolution of $M$ associated to $X$ :

$$
X^{+}=\cdots \longrightarrow X_{n} \longrightarrow X_{n-1} \longrightarrow \cdots \longrightarrow X_{1} \longrightarrow X_{0} \longrightarrow M \longrightarrow 0 .
$$


The $\mathcal{X}$-projective dimension of $M$ is the quantity

$\mathcal{X}$-pd $\operatorname{pd}_{R}(M)=\inf \left\{\sup \left\{n \geq 0 \mid X_{n} \neq 0\right\} \mid X\right.$ is an $\mathcal{X}$-resolution of $\left.M\right\}$.

In particular, one has $\mathcal{X}-\operatorname{pd}_{R}(0)=-\infty$. The modules of the $\mathcal{X}$ projective dimension 0 are the nonzero modules of $\mathcal{X}$. We set

$$
\overline{\mathcal{X}}=\text { the subcategory of } R \text {-modules with } \mathcal{X} \text { - } \operatorname{pd}_{R}(M)<\infty .
$$

An $\mathcal{X}$-resolution $X$ of $M$ is proper if the augmented resolution $X^{+}$ is $\operatorname{Hom}_{R}(\mathcal{X},-)$-exact.

We define (proper) $\mathcal{X}$-coresolution and $\mathcal{X}$-injective dimension dually. And the $\mathcal{X}$-injective dimension of $M$ is denoted $\mathcal{X}$-id ${ }_{R}(M)$.

Fact 1.6. Let ${ }_{S} C_{R}$ be a semidualizing bimodule. The Auslander class $\mathcal{A}_{C}(R)$ contains every projective and $C$-injective $R$-module, and the Bass class $\mathcal{B}_{C}(S)$ contains every injective and $C$-projective $S$-module; see [10, Lemmas 4.1 and 5.1]. These classes also satisfy the two-ofthree property by [10, Corollary 6.3]. Hence, $\mathcal{A}_{C}(R)$ contains the $R$ modules of finite projective dimension and those of finite $\mathcal{I}_{C}$-injective dimension, and $\mathcal{B}_{C}(S)$ contains the $S$-modules of finite injective dimension and those of finite $\mathcal{P}_{C}$-projective dimension. From [5, Lemma 3.9] we know that an $S$-module $M$ is in $\mathcal{B}_{C}(S)$ if and only if $\operatorname{Hom}_{S}(C, M)$ is in $\mathcal{A}_{C}(R)$, and that $M \in \mathcal{A}_{C}(R)$ if and only if $C \otimes_{R} M \in \mathcal{B}_{C}(S)$.

Let $\mathcal{C}$ be a class of modules. We define, for $i \geqslant 1$,

$$
\begin{aligned}
& { }^{\perp} \mathcal{C}=\bigcap_{i=1}^{\infty}{ }^{\perp_{i}} \mathcal{C}, \text { where }{ }^{\perp_{i}} \mathcal{C}=\left\{X \mid \operatorname{Ext}^{i}(X, C)=0 \text { for all } C \in \mathcal{C}\right\} \\
& \mathcal{C}^{\perp}=\bigcap_{i=1}^{\infty} \mathcal{C}^{\perp_{i}}, \text { where } \mathcal{C}^{\perp_{i}}=\left\{X \mid \operatorname{Ext}^{i}(C, X)=0 \text { for all } C \in \mathcal{C}\right\}
\end{aligned}
$$

2. Ding $C$-projective modules. The Ding projective modules of interest in this paper are built from semidualizing modules and their associated $C$-projective and $C$-flat classes. 
Definition 2.1. A complete $\mathcal{D}\left(\mathcal{P}_{C}\right)$-resolution is an exact sequence of $C$-projective $S$-modules

$$
X=\cdots C \otimes_{R} P_{1} \longrightarrow C \otimes_{R} P_{0} \longrightarrow C \otimes_{R} P^{0} \longrightarrow C \otimes_{R} P^{1} \longrightarrow \cdots
$$

such that $X$ is $\operatorname{Hom}_{S}\left(\mathcal{P}_{C}(S),-\right)$ - and $\operatorname{Hom}_{S}\left(-, \mathcal{F}_{C}(S)\right)$-exact.

An $S$-module $M$ is called Ding $C$-projective if there exists a complete $\mathcal{D}\left(\mathcal{P}_{C}\right)$-resolution $X$ with $M \cong \operatorname{Coker}\left(C \otimes_{R} P_{1} \rightarrow C \otimes_{R} P_{0}\right)$.

A complete $\mathcal{D}\left(\mathcal{I}_{C}\right)$-resolution is an exact sequence of $C$-injective $R$ modules

$$
\begin{aligned}
Y=\cdots \longrightarrow \operatorname{Hom}_{S}\left(C, I_{1}\right) & \longrightarrow \operatorname{Hom}_{S}\left(C, I_{0}\right) \\
& \longrightarrow \operatorname{Hom}_{S}\left(C, I^{0}\right) \longrightarrow \operatorname{Hom}_{S}\left(C, I^{1}\right) \longrightarrow \cdots
\end{aligned}
$$

such that $Y$ is $\operatorname{Hom}_{R}\left(\mathcal{F} \mathcal{I}_{C}(R),-\right)$ - and $\operatorname{Hom}_{R}\left(-, \mathcal{I}_{C}(R)\right)$-exact.

An $R$-module $N$ is called Ding $C$-injective if there exists a complete $\mathcal{D}\left(\mathcal{I}_{C}\right)$-resolution $Y$ with $N \cong \operatorname{Coker}\left(\operatorname{Hom}_{S}\left(C, I_{1}\right) \rightarrow \operatorname{Hom}_{S}\left(C, I_{0}\right)\right)$. We set

$$
\begin{aligned}
\mathcal{D}\left(\mathcal{P}_{C}\right) & =\mathcal{D}\left(\mathcal{P}_{C}(S)\right) \\
& =\text { the subcategory of Ding } C \text {-projective } S \text {-modules, } \\
\mathcal{D}\left(\mathcal{I}_{C}\right) & =\mathcal{D}\left(\mathcal{I}_{C}(R)\right) \\
& =\text { the subcategory of Ding } C \text {-injective } R \text {-modules. }
\end{aligned}
$$

Note that, when ${ }_{S} C_{R}={ }_{R} R_{R}$, the definitions above correspond to the definitions of Ding projective and Ding injective modules.

Remark 2.2. In the following, we shall only deal with the results concerning Ding $C$-projective modules. But it should be pointed out that all of the obtained results have Ding $C$-injective counterparts by using dual arguments.

\section{Fact 2.3.}

(1) It is clear that every $C$-projective $S$-module is Ding C-projective.

(2) The class $\mathcal{D}\left(\mathcal{P}_{C}\right)$ is closed under direct sums by definition.

(3) If $X=\cdots \rightarrow C \otimes_{R} P_{1} \rightarrow C \otimes_{R} P_{0} \rightarrow C \otimes_{R} P^{0} \rightarrow C \otimes_{R} P^{1} \rightarrow \cdots$ is a $\operatorname{Hom}_{S}\left(\mathcal{P}_{C}(S),-\right)$ - and $\operatorname{Hom}_{S}\left(-, \mathcal{F}_{C}(S)\right)$-exact sequence of $C$ - 
projective $S$-modules, then, by symmetry, all the images, the kernels and the cokernels of $X$ are Ding $C$-projective.

(4) By [5, Proposition 3.6], we have $\mathcal{D}\left(\mathcal{P}_{C}\right) \subseteq \mathcal{B}_{C}(S) \cap \mathcal{G P}_{C}(S)$.

Notation 2.4. Let ${ }_{S} C_{R}$ be a semidualizing bimodule. We use the following abbreviations.

$$
\begin{aligned}
\operatorname{pd}_{R}(-) & =\mathcal{P}(R)-\operatorname{pd}(-) & \operatorname{id}_{S}(-) & =\mathcal{I}(S)-\mathrm{id}(-) \\
\mathcal{P}_{C}-\operatorname{pd}_{S}(-) & =\mathcal{P}_{C}(S)-\operatorname{pd}(-) & \mathcal{I}_{C}-\mathrm{id}_{R}(-) & =\mathcal{I}_{C}(R)-\operatorname{id}(-) \\
\mathcal{F}_{C}-\operatorname{pd}_{S}(-) & =\mathcal{F}_{C}(S)-\operatorname{pd}(-) & \mathcal{F} \mathcal{I}_{C}-\mathrm{id}_{R}(-) & =\mathcal{F} \mathcal{I}_{C}(R)-\operatorname{id}(-) \\
\operatorname{Dppd}_{R}(-) & =\mathcal{D} \mathcal{P}(R)-\operatorname{pd}(-) & \operatorname{Did}_{S}(-) & =\mathcal{D} \mathcal{I}(S)-\operatorname{id}(-) \\
\mathcal{D}\left(\mathcal{P}_{C}\right)-\operatorname{pd}_{S}(-) & =\mathcal{D}\left(\mathcal{P}_{C}(S)\right)-\operatorname{pd}(-) & \mathcal{D}\left(\mathcal{I}_{C}\right)-\mathrm{id}_{R}(-) & =\mathcal{D}\left(\mathcal{I}_{C}(R)\right)-\operatorname{id}(-) .
\end{aligned}
$$

The following proposition is immediate by definition.

Proposition 2.5. An $S$-module $M$ is Ding $C$-projective if and only if:

(1) $M \in{ }^{\perp} \mathcal{F}_{C} \cap \mathcal{P}_{C}^{\perp}$,

(2) $M$ has a proper $\mathcal{P}_{C}$-resolution,

(3) $M$ has a $\operatorname{Hom}_{S}\left(-, \mathcal{F}_{C}\right)$-exact $\mathcal{P}_{C}$-coresolution.

Remark 2.6. If $L$ is an $S$-module with $\mathcal{F}_{C}-\operatorname{pd}_{S}(L)<\infty$, then $L \in \mathcal{D}\left(\mathcal{P}_{C}\right)^{\perp}$ by dimension shifting.

Theorem 2.7. The class $\mathcal{D}\left(\mathcal{P}_{C}\right)$ is closed under extensions.

Proof. Just use Proposition 2.5 and the "Horseshoe lemma" ([3, Lemma 8.2.1] and [8, (1.7)]).

Theorem 2.8. The class $\mathcal{D}\left(\mathcal{P}_{C}\right)$ is closed under direct summands.

Proof. Let $M \oplus N$ be a Ding $C$-projective $S$-module. Then there is a $\operatorname{Hom}_{S}\left(\mathcal{P}_{C},-\right)$ - and $\operatorname{Hom}_{S}\left(-, \mathcal{F}_{C}\right)$-exact sequence of $C$-projective $S$-modules

$$
X=\cdots \rightarrow C \otimes_{R} P_{1} \rightarrow C \otimes_{R} P_{0} \rightarrow C \otimes_{R} P^{0} \rightarrow C \otimes_{R} P^{1} \rightarrow \cdots
$$


with $M \oplus N=\operatorname{Coker}\left(C \otimes_{R} P_{1} \rightarrow C \otimes_{R} P_{0}\right)$. Consider the following pushout diagram:

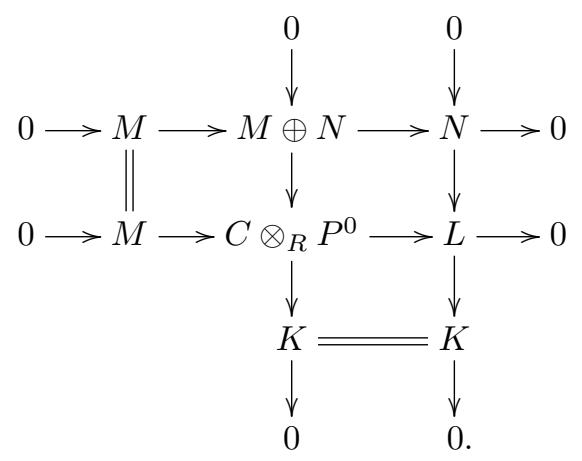

Note that the exactness of $0 \rightarrow N \rightarrow L \rightarrow K \rightarrow 0$ gives the exact sequence $0 \rightarrow M \oplus N \rightarrow M \oplus L \rightarrow K \rightarrow 0$, since $M \oplus N$ and $K$ are Ding $C$-projective, $M \oplus L$ is also Ding $C$-projective by Theorem 2.7. Thus, $L$ is also a direct summand of a Ding $C$-projective module, and so $L \in{ }^{\perp} \mathcal{F}_{C}$. By repeating the above process, we get a $\operatorname{Hom}_{S}\left(-, \mathcal{F}_{C}\right)-$ exact $\mathcal{P}_{C}$-coresolution of $M$. Dually, $M$ has a proper $\mathcal{P}_{C}$-resolution. Note that $M \oplus N \in \mathcal{P}_{C}^{\perp} \cap \perp^{\perp} \mathcal{F}_{C}$. Then $M \in \mathcal{P}_{C}^{\perp} \cap{ }^{\perp} \mathcal{F}_{C}$. Thus, $M$ is Ding $C$-projective by Proposition 2.5.

3. Ding $C$-projective dimensions of modules. In this section, we investigate some properties of the Ding $C$-projective dimensions of modules.

Proposition 3.1. Let $M$ be an $S$-module. Then $M$ has a $\mathcal{P}_{C}(S)$ resolution if and only if $M$ has a $\mathcal{D}\left(\mathcal{P}_{C}\right)$-resolution.

Proof. It is sufficient to show the "if" part. Let $0 \rightarrow N \rightarrow D_{0} \rightarrow$ $M \rightarrow 0$ be an exact sequence with $D_{0} \in \mathcal{D}\left(\mathcal{P}_{C}\right)$ and $N$ having a $\mathcal{D}\left(\mathcal{P}_{C}\right)$ resolution. Since $D_{0}$ is Ding $C$-projective, there exists a short exact sequence of $S$-modules $0 \rightarrow D^{\prime} \rightarrow C \otimes_{R} P_{0} \rightarrow D_{0} \rightarrow 0$ with $P_{0} \in \mathcal{P}(R)$ 
and $D^{\prime} \in \mathcal{D}\left(\mathcal{P}_{C}\right)$. Consider the following pullback diagram:

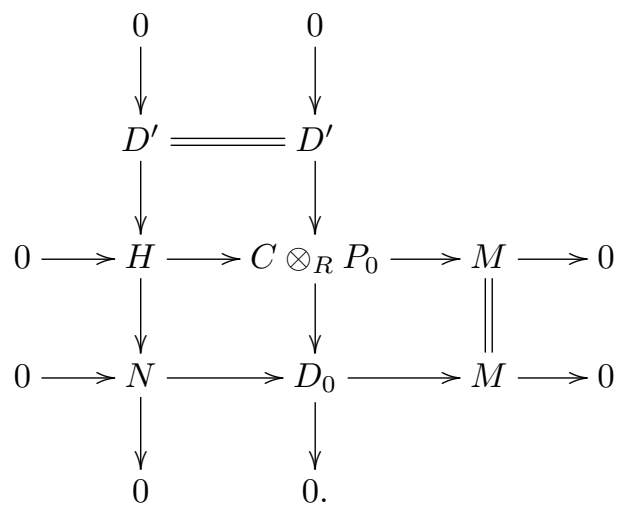

And, we have the following pullback diagram:

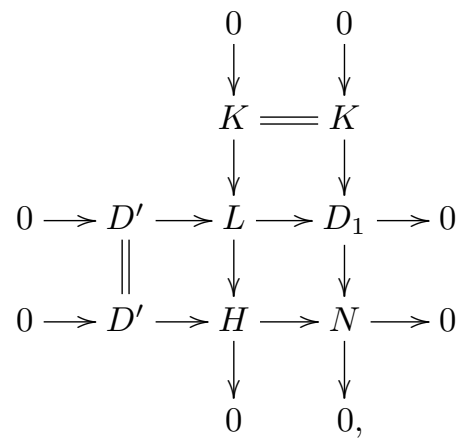

where $K$ has a $\mathcal{D}\left(\mathcal{P}_{C}\right)$-resolution and $D_{1} \in \mathcal{D}\left(\mathcal{P}_{C}\right)$. So $L \in \mathcal{D}\left(\mathcal{P}_{C}\right)$ by Theorem 2.7, and then $H$ has a $\mathcal{D}\left(\mathcal{P}_{C}\right)$-resolution. Note that $0 \rightarrow H \rightarrow C \otimes_{R} P_{0} \rightarrow M \rightarrow 0$ is exact from the first diagram. By repeating the preceding process, we have that $M$ has a $\mathcal{P}_{C}(S)$ resolution.

Remark 3.2. Let $M$ be an $S$-module with $\mathcal{D}\left(\mathcal{P}_{C}\right)-\operatorname{pd}_{S}(M)=n \geq 1$, and let $0 \rightarrow N \rightarrow D_{0} \rightarrow M \rightarrow 0$ be an exact sequence with $D_{0} \in \mathcal{D}\left(\mathcal{P}_{C}\right)$ and $\mathcal{D}\left(\mathcal{P}_{C}\right)-\operatorname{pd}_{S}(N)=n-1$. By the proof of the proposition above, we have an exact sequence $0 \rightarrow H \rightarrow C \otimes_{R} P_{0} \rightarrow M \rightarrow 0$ such that $P_{0}$ is projective and $\mathcal{D}\left(\mathcal{P}_{C}\right)-\operatorname{pd}_{S}(H)=\mathcal{D}\left(\mathcal{P}_{C}\right)-\operatorname{pd}_{S}(N)$. 
The main result of this section gives both functorial descriptions of Ding $C$-projective dimensions of modules and some criteria for computing Ding $C$-projective dimensions of modules.

Theorem 3.3. Let $M$ be an $S$-module with finite Ding C-projective dimension and $n$ a nonnegative integer. Then the following are equivalent:

(1) $\mathcal{D}\left(\mathcal{P}_{C}\right)-\operatorname{pd}_{S}(M) \leq n$.

(2) There is an exact sequence $0 \rightarrow D \rightarrow C \otimes_{R} P_{n-1} \rightarrow \cdots \rightarrow$ $C \otimes_{R} P_{1} \rightarrow C \otimes_{R} P_{0} \rightarrow M \rightarrow 0$ with $P_{i} \in \mathcal{P}(R)$ for $0 \leqslant i \leqslant n-1$ and $D \in \mathcal{D}\left(\mathcal{P}_{C}\right)$.

(3) $M$ has a proper $\mathcal{D}\left(\mathcal{P}_{C}\right)$-resolution of length $n$.

(4) There is an exact sequence $0 \rightarrow C \otimes_{R} P_{n} \rightarrow C \otimes_{R} P_{n-1} \rightarrow \cdots \rightarrow$ $C \otimes_{R} P_{1} \rightarrow D \rightarrow M \rightarrow 0$ with $P_{i} \in \mathcal{P}(R)$ for $1 \leqslant i \leqslant n$ and $D \in \mathcal{D}\left(\mathcal{P}_{C}\right)$.

(5) There is an exact sequence $0 \rightarrow C \otimes_{R} P_{n} \rightarrow \cdots \rightarrow C \otimes_{R} P_{i+1} \rightarrow$ $D \rightarrow C \otimes_{R} P_{i-1} \cdots \rightarrow C \otimes_{R} P_{0} \rightarrow M \rightarrow 0$ with $P_{j} \in \mathcal{P}(R)$ for $1 \leqslant j \leqslant n, j \neq i, 0 \leqslant i \leqslant n$ and $D \in \mathcal{D}\left(\mathcal{P}_{C}\right)$.

(6) $\operatorname{Ext}_{S}^{i}\left(M, C \otimes_{R} F\right)=0$ for all $i>n$ and all flat $R$-modules $F$.

(7) $\operatorname{Ext}_{S}^{i}(M, L)=0$ for all $i>n$ and all $S$-modules $L$ with $\mathcal{F}_{C}-\operatorname{pd}_{S}(L)<$ $\infty$.

(8) $\operatorname{Ext}_{S}^{n+1}(M, L)=0$ for all $S$-modules $L$ with $\mathcal{F}_{C}-\operatorname{pd}_{S}(L)<\infty$.

Consequently, the Ding C-projective dimension of $M$ is determined by the formulas:

$$
\begin{aligned}
\mathcal{D}\left(\mathcal{P}_{C}\right)-\operatorname{pd}_{S}(M) & =\sup \left\{i \in \mathbb{N}_{0} \mid \exists L \in \overline{\mathcal{F}_{C}(S)}: \operatorname{Ext}_{S}^{i}(M, L) \neq 0\right\} \\
& =\sup \left\{i \in \mathbb{N}_{0} \mid \exists Q \in \mathcal{F}_{C}(S): \operatorname{Ext}_{S}^{i}(M, Q) \neq 0\right\} .
\end{aligned}
$$

Proof. We first prove the equivalences of (1)-(5). The case $n=0$ is trivial. Now, we assume that $n \geq 1$.

$(1) \Rightarrow(2)$. By (1), there exists an exact sequence $0 \rightarrow N \rightarrow D_{0} \rightarrow$ $M \rightarrow 0$ with $D_{0} \in \mathcal{D}\left(\mathcal{P}_{C}\right)$ and $\mathcal{D}\left(\mathcal{P}_{C}\right)-\operatorname{pd}_{S}(N) \leq n-1$. By Remark 3.2, we have an exact sequence $0 \rightarrow H \rightarrow C \otimes_{R} P_{0} \rightarrow M \rightarrow 0$ such that $\mathcal{D}\left(\mathcal{P}_{C}\right)-\operatorname{pd}_{S}(H)=\mathcal{D}\left(\mathcal{P}_{C}\right)-\operatorname{pd}_{S}(N)$. By repeating this process, we have an exact sequence $0 \rightarrow D_{n} \rightarrow C \otimes_{R} P_{n-1} \rightarrow \cdots \rightarrow C \otimes_{R} P_{1} \rightarrow$ $C \otimes_{R} P_{0} \rightarrow M \rightarrow 0$ with $P_{i} \in \mathcal{P}(R)$ for all $0 \leqslant i \leqslant n-1$ and $D_{n} \in \mathcal{D}\left(\mathcal{P}_{C}\right)$. 
$(2) \Rightarrow(3)$. Since $D$ is Ding $C$-projective by (2), there is a $\operatorname{Hom}_{S}\left(-, \mathcal{F}_{C}(S)\right)$-exact sequence $0 \rightarrow D \rightarrow C \otimes_{R} P^{0} \rightarrow \cdots \rightarrow$ $C \otimes_{R} P^{n-1} \rightarrow D^{\prime} \rightarrow 0$ with each $P^{i} \in \mathcal{P}(R)$ and $D^{\prime} \in \mathcal{D}\left(\mathcal{P}_{C}\right)$. So the diagram:

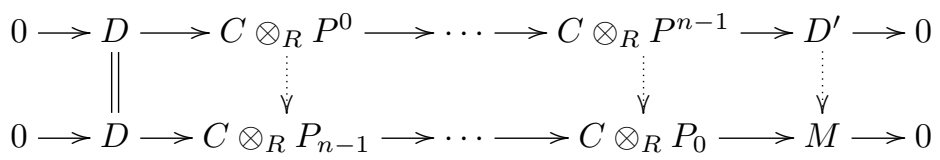

can be completed to a commutative diagram by the comparison lemma [8, Proposition 1.8]. Then the mapping cone $0 \rightarrow D \rightarrow D \oplus$ $\left(C \otimes_{R} P^{0}\right) \rightarrow C \otimes_{R}\left(P_{n-1} \oplus P^{1}\right) \rightarrow \cdots \rightarrow C \otimes_{R}\left(P_{1} \oplus P^{n-1}\right) \rightarrow$ $\left(C \otimes_{R} P_{0}\right) \oplus D^{\prime} \rightarrow M \rightarrow 0$ gives an exact sequence $X_{\bullet}=0 \rightarrow C \otimes_{R} P^{0} \rightarrow$ $C \otimes_{R}\left(P_{n-1} \oplus P^{1}\right) \rightarrow \cdots \rightarrow C \otimes_{R}\left(P_{1} \oplus P^{n-1}\right) \rightarrow\left(C \otimes_{R} P_{0}\right) \oplus D^{\prime} \rightarrow$ $M \rightarrow 0$. Note that each cokernel of $X_{\text {• }}$ except $M$ has a finite $\mathcal{P}_{C}(S)$ resolution. So $X_{\bullet}$ is $\operatorname{Hom}_{S}\left(\mathcal{D}\left(\mathcal{P}_{C}\right),-\right.$ )-exact by Remark 2.6. It follows that $X_{\bullet}$ is just a proper $\mathcal{D}\left(\mathcal{P}_{C}\right)$-resolution of $M$ of length $n$.

$(3) \Rightarrow(1),(4) \Rightarrow(1)$ and $(5) \Rightarrow(1)$ are trivial.

$(2) \Rightarrow(4)$. Note that $X_{\bullet}$ in the proof of $(2) \Rightarrow(3)$ is just the desired exact sequence.

$(1) \Rightarrow(5)$ is immediate by Remark 3.2 , and the equivalence of (1) and (4).

Next we show the equivalences of (1), (6), (7) and (8).

$(1) \Rightarrow(6)$. By hypothesis, there exists an exact sequence $0 \rightarrow D_{n} \rightarrow$ $\cdots \rightarrow D_{1} \rightarrow D_{0} \rightarrow M \rightarrow 0$ with $D_{i} \in \mathcal{D}\left(\mathcal{P}_{C}\right)$ for $0 \leqslant i \leqslant n$. So $\operatorname{Ext}_{S}^{n+j}\left(M, C \otimes_{R} F\right) \cong \operatorname{Ext}_{S}^{j}\left(D_{n}, C \otimes_{R} F\right)=0$ for all $j>0$ and all flat $R$-modules $F$ by Proposition 2.5.

$(6) \Rightarrow(7)$ by the usual dimension shifting argument.

$(7) \Rightarrow(8)$ is clear.

$(8) \Rightarrow(1)$. By assumption, let $\mathcal{D}\left(\mathcal{P}_{C}\right)-\operatorname{pd}_{S}(M)=s<\infty$. If $s \leqslant n$, there is nothing to prove. So we assume $s>n$. Then there is an exact sequence $0 \rightarrow D_{s} \rightarrow \cdots \rightarrow D_{1} \rightarrow D \rightarrow M \rightarrow 0$ with $D_{i} \in \mathcal{P}_{C}(S)$ for $1 \leqslant i \leqslant s$ and $D \in \mathcal{D}\left(\mathcal{P}_{C}\right)$ by the equivalence of (1) and (4). Let $K_{i}=\operatorname{Coker}\left(D_{i+1} \rightarrow D_{i}\right)$ for $1 \leqslant i \leqslant s-1$.

If $n=0$, then $\operatorname{Ext}_{S}^{n+1}\left(M, K_{1}\right)=0$ by (8) since $\mathcal{F}_{C}-\operatorname{pd}_{S}\left(K_{1}\right)<\infty$. Thus, the exact sequence $0 \rightarrow K_{1} \rightarrow D \rightarrow M \rightarrow 0$ splits, and so 
$M \in \mathcal{D}\left(\mathcal{P}_{C}\right)$ by Theorem 2.8 , as desired.

Let $n \geqslant 1$. Since $\mathcal{F}_{C}-\operatorname{pd}_{S}\left(K_{n+1}\right)<\infty$, we have that $\operatorname{Ext}_{S}^{1}\left(K_{n}, K_{n+1}\right)$ $\cong \operatorname{Ext}_{S}^{n+1}\left(M, K_{n+1}\right)=0$ by (8). So the exact sequence $0 \rightarrow K_{n+1} \rightarrow$ $D_{n} \rightarrow K_{n} \rightarrow 0$ splits, and then $K_{n} \in \mathcal{P}_{C}(S)$. Hence, (1) follows.

The last formulas in the theorem for determination of $\mathcal{D}\left(\mathcal{P}_{C}\right)-\operatorname{pd}_{S}(M)$ are a direct consequence of the equivalences between (1), (6) and (7).

Let $\mathcal{X}$ be any class of $R$-modules and $M$ an $R$-module. An $\mathcal{X}$ precover of $M$ is an $R$-homomorphism $\varphi: X \rightarrow M$, where $X \in \mathcal{X}$ and such that the sequence $\operatorname{Hom}_{R}\left(X^{\prime}, X\right) \stackrel{\operatorname{Hom}_{R}\left(X^{\prime}, \varphi\right)}{\longrightarrow} \operatorname{Hom}_{R}\left(X^{\prime}, M\right) \rightarrow 0$ is exact for every $X^{\prime} \in \mathcal{X}$.

From the equivalence of (1) and (3) in Theorem 3.3, we immediately have the following:

Corollary 3.4. Let $M$ be an $S$-module with $\mathcal{D}\left(\mathcal{P}_{C}\right)-\operatorname{pd}_{S}(M)=n<\infty$. Then there is a surjective $\mathcal{D}\left(\mathcal{P}_{C}\right)$-precover $\varphi: N \rightarrow M$ such that $K=\operatorname{Ker} \varphi$ satisfies $\mathcal{P}_{C}-\operatorname{pd}_{S}(K)=n-1$ (if $n=0$, this should be interpreted as $K=0$ ).

The following result is the Ding $C$-projective version of $[\mathbf{1}$, Lemma 2.17].

Corollary 3.5. Let $M$ be an $S$-module with $\mathcal{D}\left(\mathcal{P}_{C}\right)-\operatorname{pd}_{S}(M)=n<\infty$. Then there exists an exact sequence $0 \rightarrow M \rightarrow H \rightarrow D \rightarrow 0$, where $D$ is Ding C-projective and $\mathcal{P}_{C}-\operatorname{pd}_{S}(H)=n$.

Proof. If $M$ is Ding $C$-projective, we take $0 \rightarrow M \rightarrow H \rightarrow D \rightarrow 0$ to be the first short exact sequence in the "right half" of a complete $\mathcal{D}\left(\mathcal{P}_{C}\right)$-resolution of $M$.

Now assume that $\mathcal{D}\left(\mathcal{P}_{C}\right)-\operatorname{pd}_{S}(M)=n>0$. By Corollary 3.4, there is an exact sequence $0 \rightarrow K \rightarrow D^{\prime} \rightarrow M \rightarrow 0$, where $D^{\prime} \in \mathcal{D}\left(\mathcal{P}_{C}\right)$ and $\mathcal{P}_{C}-\operatorname{pd}_{S}(K)=n-1$. Since $D^{\prime}$ is Ding $C$-projective, there exists an exact sequence $0 \rightarrow D^{\prime} \rightarrow C \otimes_{R} P \rightarrow D \rightarrow 0$ such that $P \in \mathcal{P}(R)$ and 
$D \in \mathcal{D}\left(\mathcal{P}_{C}\right)$. Consider the pushout diagram:

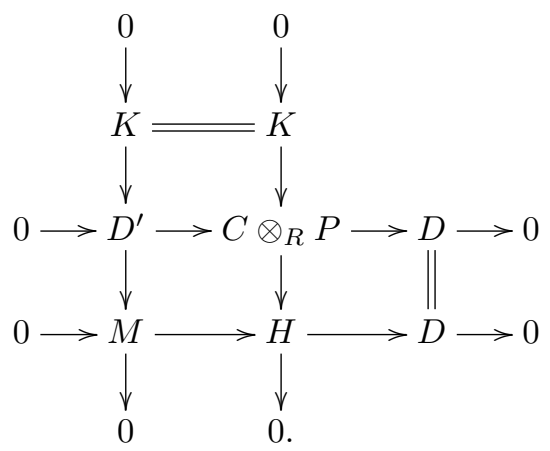

The bottom row of this diagram is the desired sequence. To see this, we must argue that $\mathcal{P}_{C}-\operatorname{pd}_{S}(H)=n$. Clearly, $\mathcal{P}_{C}-\operatorname{pd}_{S}(H) \not \leq n-1$. If $n=1$, then we must have $\mathcal{P}_{C}-\operatorname{pd}_{S}(H)=1$; otherwise, $M \in \mathcal{D}\left(\mathcal{P}_{C}\right)$ by Corollary 3.4. If $n>1$, from the short exact sequence $0 \rightarrow K \rightarrow$ $C \otimes_{R} P \rightarrow H \rightarrow 0$, we have $\mathcal{P}_{C}-\operatorname{pd}_{S}(H)=\mathcal{P}_{C}-\operatorname{pd}_{S}(K)+1=n$ since $\mathcal{P}_{C}-\operatorname{pd}_{S}(K) \neq \mathcal{P}_{C}-\operatorname{pd}_{S}\left(C \otimes_{R} P\right)$. Then, in conclusion, we have $\mathcal{P}_{C}-\operatorname{pd}_{S}(H)=n$.

Corollary 3.6. Let $0 \rightarrow M^{\prime} \rightarrow M \rightarrow M^{\prime \prime} \rightarrow 0$ be an exact sequence of $S$-modules with $M \in \mathcal{D}\left(\mathcal{P}_{C}\right)$.

(1) If $M^{\prime} \in \mathcal{D}\left(\mathcal{P}_{C}\right)$, then $M^{\prime \prime} \in \mathcal{D}\left(\mathcal{P}_{C}\right)$ if and only if $M^{\prime \prime} \in{ }^{\perp_{1}} \mathcal{F}_{C}$.

(2) If $M^{\prime \prime} \in \mathcal{D}\left(\mathcal{P}_{C}\right)$, then $M^{\prime} \in \mathcal{D}\left(\mathcal{P}_{C}\right)$ if and only if $M^{\prime} \in \mathcal{P}_{C}^{\perp_{1}}$.

Proof. (1) The necessity follows from Proposition 2.5. We now prove the sufficiency. Since $\mathcal{D}\left(\mathcal{P}_{C}\right)-\operatorname{pd}_{S}\left(M^{\prime \prime}\right) \leq 1$, by Corollary 3.4, there is an exact sequence $0 \rightarrow C \otimes_{R} P \rightarrow D \rightarrow M^{\prime \prime} \rightarrow 0$, where $P \in \mathcal{P}(R)$ and $D \in \mathcal{D}\left(\mathcal{P}_{C}\right)$. By assumption $\operatorname{Ext}_{S}^{1}\left(M^{\prime \prime}, C \otimes_{R} P\right)=0$, this sequence splits, and hence $M^{\prime \prime}$ is Ding $C$-projective by Theorem 2.8.

(2) The necessity follows from Proposition 2.5. Since $M^{\prime \prime} \in \mathcal{D}\left(\mathcal{P}_{C}\right)$, there is an exact sequence $0 \rightarrow K \rightarrow C \otimes_{R} P_{0} \rightarrow M^{\prime \prime} \rightarrow 0$ with 
$P_{0} \in \mathcal{P}(R)$ and $K \in \mathcal{D}\left(\mathcal{P}_{C}\right)$. Consider the following pullback diagram:

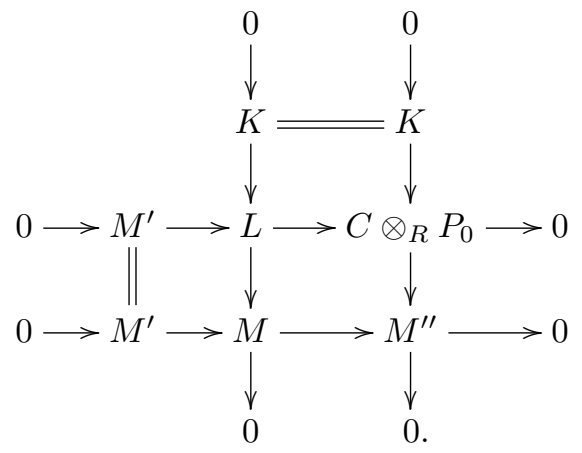

From the middle column, we get that $L$ is Ding $C$-projective by Theorem 2.7. Note that the middle row splits since $\operatorname{Ext}_{S}^{1}\left(C \otimes_{R}\right.$ $\left.P_{0}, M^{\prime}\right)=0$ by hypothesis, and hence, $M^{\prime}$ is Ding $C$-projective by Theorem 2.8.

Recall that a class of modules is called projectively resolving if it is closed under extensions, kernels of surjections and it contains all projective modules. By Corollary 3.6, we get that the class of Ding projective modules is projectively resolving, which was proved in $[\mathbf{1 8}$, Theorem 2.6] and [12, Theorem 2.1].

When ${ }_{S} C_{R}={ }_{R} R_{R}$, it was proved in [2, Lemma 2.4 (2)] that, if $M$ is a Ding projective $R$-module of finite flat dimension, then $M$ is projective. Naturally, it makes sense to give the relation between $\mathcal{P}_{C^{-}}$ projective dimensions and $\mathcal{D}\left(\mathcal{P}_{C}\right)$-projective dimensions.

Proposition 3.7. If $M$ is an $S$-module of finite $\mathcal{F}_{C}$-projective dimension, then

$$
\mathcal{D}\left(\mathcal{P}_{C}\right)-\operatorname{pd}_{S}(M)=\mathcal{P}_{C}-\operatorname{pd}_{S}(M) .
$$

In particular, there is an equality of classes $\mathcal{D}\left(\mathcal{P}_{C}\right) \cap \overline{\mathcal{F}_{C}(S)}=\mathcal{P}_{C}(S)$.

Proof. Using Theorem 3.3, it suffices to show that if $M$ is Ding $C$ projective with $\mathcal{F}_{C}-\operatorname{pd}_{S}(M)<\infty$, then $M$ is $C$-projective. To this end, consider an exact sequence of the form

$$
0 \longrightarrow K \longrightarrow C \otimes_{R} P \longrightarrow M \longrightarrow 0,
$$


where $P \in \mathcal{P}(R)$ and $\mathcal{F}_{C}-\operatorname{pd}_{S}(K)<\infty$. By Theorem 3.3, $\operatorname{Ext}_{\bar{S}}^{\geq 1}(M, K)$ $=0$, so the above sequence splits, forcing $M$ to be a summand of $C \otimes_{R} P$. Since the class of $C$-projectives is closed under summands by [10, Proposition 5.5], $M$ is $C$-projective, as desired.

4. The Foxby equivalence. In this section, we will study the Foxby equivalence between some subclasses of the Auslander class $\mathcal{A}_{C}(R)$ and the Bass class $\mathcal{B}_{C}(S)$.

Lemma 4.1. If $M \in \mathcal{B}_{C}(S)$, then $M$ is Ding $C$-projective if and only if $\operatorname{Hom}_{S}(C, M)$ is Ding projective.

Proof. $\Rightarrow$. By Proposition 2.5, there exist projective $R$-modules $P^{0}, P^{1}, \ldots$ together with an exact sequence

$$
0 \longrightarrow M \longrightarrow C \otimes_{R} P^{0} \longrightarrow C \otimes_{R} P^{1} \longrightarrow \cdots .
$$

Furthermore, the complex $\operatorname{Hom}_{S}\left((*), C \otimes_{R} F\right)$ is exact for all flat $R$ modules $F$.

Since $M$ and each $C \otimes_{R} P^{i}$ are in $\mathcal{B}_{C}(S)$, by [10, Theorem 1], the definition of $\mathcal{B}_{C}(S)$ gives that both $\operatorname{Ext}_{\bar{S}}^{\geq 1}(C, M)=0$ and $\operatorname{Ext}_{\bar{S}}^{\geq 1}\left(C, C \otimes_{R} P^{i}\right)=0$ for all $i \geq 0$. Hence, the sequence $(*)$ stays exact if we apply to it the functor $\operatorname{Hom}_{S}(C,-)$, and doing so we obtain the exact sequence of $R$-modules

$$
0 \longrightarrow \operatorname{Hom}_{S}(C, M) \longrightarrow P^{0} \longrightarrow P^{1} \longrightarrow \cdots .
$$

By similar arguments, we see that if we apply $C \otimes_{R}-$ to the sequence $(* *)$, then we have $(*)$ back. Let $F$ be any flat $R$-module. Then $F \cong \operatorname{Hom}_{S}\left(C, C \otimes_{R} F\right)$ since $F \in \mathcal{A}_{C}(R)$. So we have:

$$
\begin{aligned}
\operatorname{Hom}_{R}((* *), F) & \cong \operatorname{Hom}_{R}\left((* *), \operatorname{Hom}_{S}\left(C, C \otimes_{R} F\right)\right) \\
& \cong \operatorname{Hom}_{S}\left(C \otimes_{R}(* *), C \otimes_{R} F\right) \\
& \cong \operatorname{Hom}_{S}\left((*), C \otimes_{R} F\right)
\end{aligned}
$$

is exact. Thus, $(* *)$ is a $\operatorname{Hom}_{R}(-, \mathcal{F}(R))$-exact projective-coresolution of $\operatorname{Hom}_{S}(C, M)$.

Next, we claim that $\operatorname{Ext}_{\bar{R}}^{\geq 1}\left(\operatorname{Hom}_{S}(C, M), F\right)=0$ for all flat $R$ modules $F$. To this end, let $\mathbb{P}$. $\rightarrow M$ be a proper $\mathcal{P}_{C}$-projective 
resolution of $M$. Then $\operatorname{Hom}_{S}(C, \mathbb{P}$.) is a proper projective resolution of $\operatorname{Hom}_{S}(C, M)$ by [15, Lemma 2.1]. For every $i>0$, we have:

$$
\begin{aligned}
\operatorname{Ext}_{R}^{i}\left(\operatorname{Hom}_{S}(C, M), F\right) & \stackrel{(a)}{=} \mathrm{H}^{i}\left(\operatorname{Hom}_{R}\left(\operatorname{Hom}_{S}(C, \mathbb{P} .), \operatorname{Hom}_{S}\left(C, C \otimes_{R} F\right)\right)\right) \\
& \stackrel{(b)}{\cong} \mathrm{H}^{i}\left(\operatorname{Hom}_{S}\left(C \otimes_{R} \operatorname{Hom}_{S}(C, \mathbb{P} .), C \otimes_{R} F\right)\right) \\
& \stackrel{(c)}{=} \mathrm{H}^{i}\left(\operatorname{Hom}_{S}\left(\mathbb{P} ., C \otimes_{R} F\right)\right) \\
& \stackrel{(d)}{=} \operatorname{Ext}_{\mathcal{P}_{C}}^{i}\left(M, C \otimes_{R} F\right) \\
& \stackrel{(e)}{\cong} \operatorname{Ext}_{S}^{i}\left(M, C \otimes_{R} F\right) \\
& =0 .
\end{aligned}
$$

Here (a) and (c) follow from $F \in \mathcal{A}_{C}(R)$ and $\mathbb{P} . \in \mathcal{B}_{C}(S)$ (see $[\mathbf{1 0}$, Lemma 4.1; Theorem 1]); (b) is by adjointness, (d) is by the definition of relative cohomology modules [15, Lemma (1.5)] and (e) follows from [15, Corollary 4.2]. Therefore, we have proved that $\operatorname{Hom}_{S}(C, M)$ is a Ding projective $R$-module by [12, Proposition 2.1].

$\Leftarrow$. Assume that $\operatorname{Hom}_{S}(C, M)$ is a Ding projective $R$-module. It follows from [12, Proposition 2.1] that there exist projective $R$-modules $P^{0}, P^{1}, \ldots$ together with an exact sequence

$$
0 \longrightarrow \operatorname{Hom}_{S}(C, M) \longrightarrow P^{0} \longrightarrow P^{1} \longrightarrow \cdots .
$$

Furthermore, the above exact sequence is $\operatorname{Hom}_{R}(-, \mathcal{F}(R))$-exact.

Since $P^{0}, P^{1}, \ldots$ and $\operatorname{Hom}_{S}(C, M)$ are in $\mathcal{A}_{C}(R)$, it follows from [10, Corollary 6.3] that every cokernel in $(+)$ is also in $\mathcal{A}_{C}(R)$. If $N \in \mathcal{A}_{C}(R)$, then $\operatorname{Tor}_{\geq 1}^{R}(C, N)=0$, and consequently, the sequence $(+)$ stays exact when we apply to it the functor $C \otimes_{R}-$, and we have the following exact sequence:

$$
0 \longrightarrow M \longrightarrow C \otimes_{R} P^{0} \longrightarrow C \otimes_{R} P^{1} \longrightarrow \cdots .
$$

Let $F$ be any flat $R$-module. Then $F \cong \operatorname{Hom}_{S}\left(C, C \otimes_{R} F\right)$ since $F \in \mathcal{A}_{C}(R)$. Now by adjointness, we see that

$$
\begin{aligned}
\operatorname{Hom}_{S}\left((\sharp), C \otimes_{R} F\right) & \cong \operatorname{Hom}_{S}\left(C \otimes_{R}(+), C \otimes_{R} F\right) \\
& \cong \operatorname{Hom}_{R}\left((+), \operatorname{Hom}_{S}\left(C, C \otimes_{R} F\right)\right) \\
& \cong \operatorname{Hom}_{R}((+), F)
\end{aligned}
$$


is exact. Furthermore, from the first part of this proof, for any flat $R$-module $F$, we have

$$
\operatorname{Ext}_{\bar{S}}^{\geq 1}\left(M, C \otimes_{R} F\right) \cong \operatorname{Ext}_{\bar{R}}^{\geq 1}\left(\operatorname{Hom}_{S}(C, M), F\right)=0 .
$$

Dually, we can prove that $M$ has a proper $\mathcal{P}_{C}$-resolution and $M \in \mathcal{P} \perp$. Hence, $M$ is Ding $C$-projective by Proposition 2.5 .

Proposition 4.2. There are equivalences of categories:

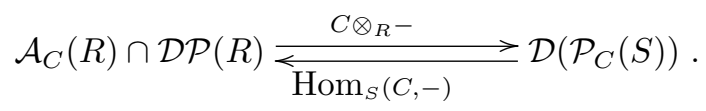

Proof. By Fact 2.3 (4), Fact 1.6 and Lemma 4.1, it is obvious that the functor $\operatorname{Hom}_{S}(C,-)$ maps $\mathcal{D}\left(\mathcal{P}_{C}(S)\right)$ to $\mathcal{A}_{C}(R) \cap \mathcal{D P}(R)$. So it suffices to show that the functor $C \otimes_{R}-$ maps $\mathcal{A}_{C}(R) \cap \mathcal{D P}(R)$ to $\mathcal{D}\left(\mathcal{P}_{C}(S)\right)$. To this end, let $M \in \mathcal{A}_{C}(R) \cap \mathcal{D P}(R)$. Then there exists a $\operatorname{Hom}_{R}(-, \mathcal{F}(R))$-exact sequence of $R$-modules

$$
\mathbb{P}=\cdots \stackrel{\partial_{2}}{\longrightarrow} P_{1} \stackrel{\partial_{1}}{\longrightarrow} P_{0} \stackrel{\partial_{0}}{\longrightarrow} P^{0} \stackrel{\partial^{0}}{\longrightarrow} P^{1} \stackrel{\partial^{1}}{\longrightarrow} \cdots
$$

with all $P_{i}, P^{i} \in \mathcal{P}(R)$ and $M \cong \operatorname{Im} \partial_{0}$. By [17, Lemma 4.1, Corollary 6.3] and [2, Remark 2.2(3)], every kernel and cokernel in $\mathbb{P}$ are in $\mathcal{A}_{C}(R) \cap \mathcal{D P}(R)$. Then, applying the functor $C \otimes_{R}-$ to it, we have the following exact sequence of $S$-modules

$$
\begin{aligned}
C \otimes_{R} \mathbb{P}=\cdots \stackrel{1_{C} \otimes_{R} \partial_{2}}{\longrightarrow} C \otimes_{R} P_{1} \stackrel{\stackrel{1_{C} \otimes_{R} \partial_{1}}{\longrightarrow} C \otimes_{R} P_{0}}{ } \\
\stackrel{1_{C} \otimes_{R} \partial_{0}}{\longrightarrow} C \otimes_{R} P^{0} \stackrel{1_{C} \otimes_{R} \partial^{0}}{\longrightarrow} \cdots .
\end{aligned}
$$

Clearly, $C \otimes_{R} M \cong \operatorname{Im}\left(1_{C} \otimes_{R} \partial_{0}\right)$. Now by [10, Theorem 6.4], for any flat $R$-module $F$, we see that

$$
\begin{aligned}
\operatorname{Hom}_{S}\left(C \otimes_{R} \mathbb{P}, C \otimes_{R} F\right) \cong & \operatorname{Hom}_{R}\left(\operatorname{Hom}_{S}\left(C, C \otimes_{R} \mathbb{P}\right),\right. \\
& \left.\operatorname{Hom}_{S}\left(C, C \otimes_{R} F\right)\right) \\
\cong & \operatorname{Hom}_{R}(\mathbb{P}, F)
\end{aligned}
$$


is exact. And, for any projective $R$-module $P$, we have

$$
\begin{aligned}
\operatorname{Hom}_{S}\left(C \otimes_{R} Q, C \otimes_{R} \mathbb{P}\right) \cong & \operatorname{Hom}_{R}\left(\operatorname{Hom}_{S}\left(C, C \otimes_{R} Q\right),\right. \\
& \left.\operatorname{Hom}_{S}\left(C, C \otimes_{R} \mathbb{P}\right)\right) \\
\cong & \operatorname{Hom}_{R}(Q, \mathbb{P})
\end{aligned}
$$

is exact. So $C \otimes_{R} \mathbb{P}$ is a $\operatorname{Hom}_{S}\left(\mathcal{P}_{C},-\right)$ - and $\operatorname{Hom}_{S}\left(-, \mathcal{F}_{C}\right)$-exact sequence, and hence $C \otimes_{R} M \in \mathcal{D}\left(\mathcal{P}_{C}(S)\right)$.

Finally, note that if $M \in \mathcal{A}_{C}(R) \cap \mathcal{D P}(R)$ and $N \in \mathcal{D}\left(\mathcal{P}_{C}(S)\right)$, then there exist natural isomorphisms $M \cong \operatorname{Hom}_{S}\left(C, C \otimes_{R} M\right)$ and $N \cong C \otimes_{R} \operatorname{Hom}_{S}(C, N)$. Then the desired equivalences of categories follow.

Let $n$ be a nonnegative integer. In the following, we denote by $\mathcal{D P}(R)_{\leq n}\left(\mathcal{D}\left(\mathcal{P}_{C}(S)\right)_{\leq n}\right)$ the class of modules with Ding projective (Ding $C$-projective) dimension at most $n$.

Theorem 4.3. There are equivalences of categories:

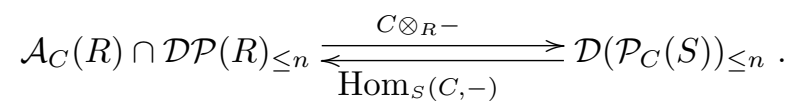

Proof. Let $M \in \mathcal{A}_{C}(R) \cap \mathcal{D P}(R)_{\leq n}$. If $n=0$, then $C \otimes_{R} M \in$ $\mathcal{D}\left(\mathcal{P}_{C}(S)\right)$ by Proposition 4.2. Now assume $n \geq 1$. Then by [12, Theorem 2.4], there exists an exact sequence of $R$-modules

$$
0 \rightarrow D_{n} \rightarrow P_{n-1} \rightarrow \cdots \rightarrow P_{1} \rightarrow P_{0} \rightarrow M \rightarrow 0
$$

with $D_{n} \in \mathcal{D P}(R)$ and $P_{i} \in \mathcal{P}(R)$ for $0 \leq i \leq n-1$. By [10, Theorem 1, Corollary 6.3], every term and kernel in the above sequence are in $\mathcal{A}_{C}(R)$. So we have the following exact sequence of $S$-modules

$$
0 \rightarrow C \otimes_{R} D_{n} \rightarrow \cdots \rightarrow C \otimes_{R} P_{1} \rightarrow C \otimes_{R} P_{0} \rightarrow C \otimes_{R} M \rightarrow 0
$$

with $C \otimes_{R} D_{n}$ and all $C \otimes_{R} P_{i}$ in $\mathcal{D}\left(\mathcal{P}_{C}(S)\right)$ by Proposition 4.2, which means that $C \otimes_{R} M \in \mathcal{D}\left(\mathcal{P}_{C}(S)\right)_{\leq n}$.

Conversely, let $N \in \mathcal{D}\left(\mathcal{P}_{C}(S)\right)_{\leq n}$. If $n=0$, then $\operatorname{Hom}_{S}(C, N) \in$ $\mathcal{A}_{C}(R) \cap \mathcal{D P}(R)$ by Proposition 4.2. Now assume $n \geq 1$. Then by Theorem 3.3, there exists an exact sequence of $S$-modules

$$
0 \rightarrow C \otimes_{R} P_{n} \rightarrow C \otimes_{R} P_{n-1} \rightarrow \cdots \rightarrow C \otimes_{R} P_{1} \rightarrow D_{0} \rightarrow N \rightarrow 0
$$


with $D_{0} \in \mathcal{D}\left(\mathcal{P}_{C}(S)\right)$ and $P_{i} \in \mathcal{P}(R)$ for $1 \leq i \leq n$. By [10, Corollaries $6.1,6.3]$, every term and cokernel in the above sequence are in $\mathcal{B}_{C}(S)$. So, applying the functor $\operatorname{Hom}_{S}(C,-)$ to it, we have the following exact sequence of $R$-modules

$$
0 \rightarrow P_{n} \rightarrow P_{n-1} \rightarrow \cdots \rightarrow P_{1} \rightarrow \operatorname{Hom}_{S}\left(C, D_{0}\right) \rightarrow \operatorname{Hom}_{S}(C, N) \rightarrow 0
$$

with $\operatorname{Hom}_{S}\left(C, D_{0}\right) \in \mathcal{A}_{C}(R) \cap \mathcal{D} \mathcal{P}(R)$ by Proposition 4.2. Hence, $\operatorname{Hom}_{S}(C, N) \in \mathcal{A}_{C}(R) \cap \mathcal{D} \mathcal{P}(R)_{\leq n}$.

The rest of the proof is similar to that of Proposition 4.2.

By assembling the information above and [11, Theorem 4.6], we have the following extension of the Foxby equivalence from the introduction.

Theorem 4.4. (Foxby equivalence). Let $n$ be a nonnegative integer, and let $\mathcal{P}(R)_{\leq n}, \mathcal{P}_{C}(S)_{\leq n}, \mathcal{I}(S)_{\leq n}$ and $\mathcal{I}_{C}(R)_{\leq n}$ be the classes of modules with projective, $C$-projective, injective and $C$-injective dimension at most $n$, respectively. Then there are equivalences of categories:

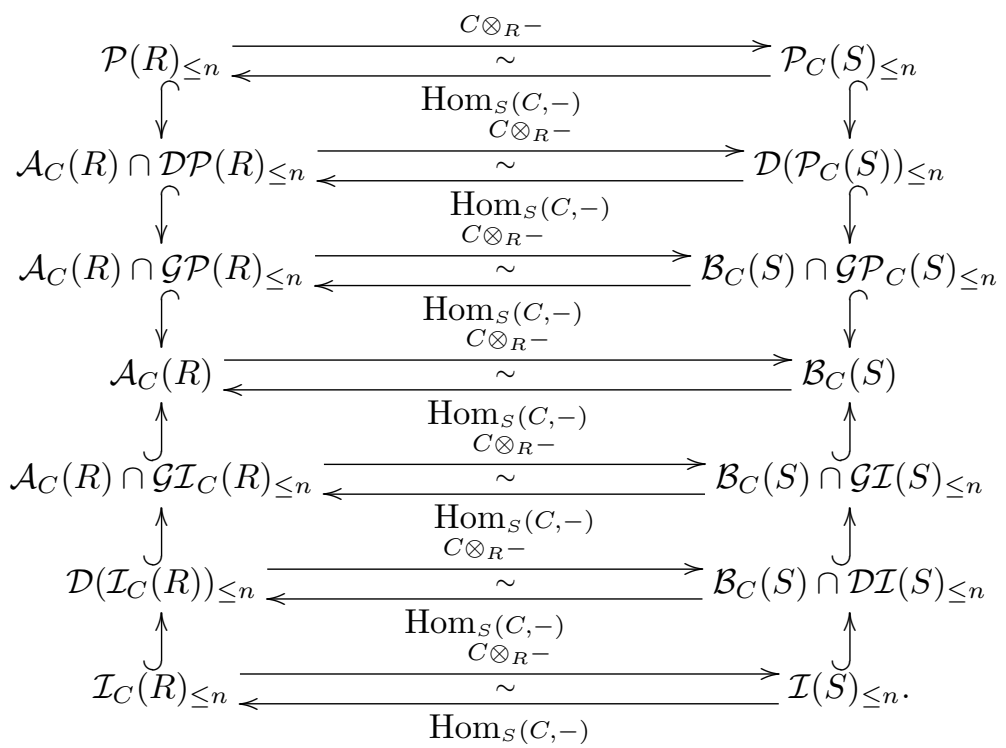

Remark 4.5. By the definition of $G_{C}$-projective modules, one can define $D_{C}$-projective modules over a commutative ring $S$ as follows: 
An $S$-module $M$ is called $D_{C}$-projective if there exists an exact sequence of $S$-modules

$$
X=\cdots \rightarrow P_{1} \rightarrow P_{0} \rightarrow C \otimes_{S} P^{0} \rightarrow C \otimes_{S} P^{1} \rightarrow \cdots
$$

with $P_{i}, P^{i}$ projective for $i \geq 0$, such that $M \cong \operatorname{Coker}\left(P_{1} \rightarrow P_{0}\right)$ and $X$ is $\operatorname{Hom}_{S}\left(-, \mathcal{F}_{C}(S)\right)$-exact. Set

$$
\mathcal{D} \mathcal{P}_{C}(S)=\text { the subcategory of } D_{C} \text {-projective } S \text {-modules. }
$$

By Proposition 2.5, we have that $\mathcal{D}\left(\mathcal{P}_{C}(S)\right)=\mathcal{B}_{C}(S) \cap \mathcal{D} \mathcal{P}_{C}(S)$.

Acknowledgments. The authors would like to thank the referees for helpful suggestions and corrections that have improved this article.

\section{REFERENCES}

1. L.W. Christensen, A. Frankild and H. Holm, On Gorenstein projective, injective and flat dimensions-a functorial description with applications, J. Alg. 302 (2006), 231-279.

2. N. Ding, Y. Li and L. Mao, Strongly Gorenstein flat modules, J. Aust. Math. Soc. 86 (2009), 323-338.

3. E.E. Enochs and O.M.G. Jenda, Relative homological algebra, de Gruyter Expo. Math. 30, Walter de Gruyter Co., Berlin, 2000.

4. H.-B. Foxby, Gorenstein modules and related modules, Math. Scand. 31 (1973), 267-284.

5. Y. Geng and N. Ding, W-Gorenstein modules, J. Alg. 325 (2011), 132-146.

6. J. Gillespie, Model structures on modules over Ding-Chen rings, Homol. Homot. Appl. 12 (2010), 61-73.

7. E.S. Golod, $G$-dimension and generalized perfect ideals, Alg. Geom. Appl. 165 (1984), 62-66.

8. H. Holm, Gorenstein homological dimensions, J. Pure Appl. Alg. 189 (2004), $167-193$.

9. H. Holm and P. Jørgensen, Semi-dualizing modules and related Gorenstein homological dimensions, J. Pure Appl. Alg. 205 (2006), 423-445.

10. H. Holm and D. White, Foxby equivalence over associative rings, J. Math. Kyoto Univ. 47 (2007), 781-808.

11. Z. Liu, Z. Huang and $\mathrm{A}$. $\mathrm{Xu}$, Gorenstein projective dimension relative to a semidualizing bimodule, Comm. Alg. 41 (2013), 1-18.

12. N. Mahdou and M. Tamekkante, Strongly Gorenstein flat modules and dimensions, Chin. Ann. Math. 32 (2011), 533-548.

13. L. Mao and N. Ding, Gorenstein FP-injective and Gorenstein flat modules, J. Alg. Appl. 7 (2008), 491-506. 
14. J.J. Rotman, An introductions to homological algebra, Academic Press, New York, 1979.

15. R. Takahashi and D. White, Homological aspects of semidualizing modules, Math. Scand. 106 (2010), 5-22.

16. W.V. Vasconcelos, Divisor theory in module categories, North-Holland Publishing Co., Amsterdam, 1974.

17. D. White, Gorenstein projective dimension with respect to a semidualizing module, J. Comm. Alg. 2 (2010), 111-137.

18. G. Yang, Z. Liu and L. Liang, Ding projective and Ding injective modules, Alg. Colloq. 20 (2013), 601-612.

Department of Mathematics, Northwest Normal University, Lanzhou, 730070, P.R. CHINA

Email address: zhangcx@nwnu.edu.cn

Department of Mathematics, Northwest Normal University, Lanzhou, 730070, P.R. CHINA

Email address: wanglm@nwnu.edu.cn

Department of Mathematics, Northwest Normal University, Lanzhou, 730070, P.R. CHINA

Email address: liuzk@nwnu.edu.cn 